\title{
Electrophysiological Study of Thalamo-Cortical Relation in
} Man

\begin{tabular}{|l|l|l|}
\hline K. & & Matsumoto \\
\hline T. & & Miyamoto \\
\hline Y. & & Mimura \\
\hline H. & & Beck \\
\hline
\end{tabular}

Department of Neurological Surgery, Okayama University Medical School, Okayama

\section{Abstract}

Single or repetitive stimulation of the thalamic nucleus has been performed during stereotaxic surgery for over 250 patients with extrapyramidal disorders or intractable pain in our service for the last 7 years, because the stimulation has been encountered as a useful tool for determination of the stereotaxic target. Aside from such direct therapeutic considerations, computer analysis of the cortical evoked responses has brought us much interesting information regarding the physiological mechanism of the thalamo-cortical relations in man. Thus, bilateral, three-negative, cortically evoked responses after single stimulation of the unilateral VL nucleus of the thalamus and differences of augmenting and recruiting responses between the human and the laboratory animal have been reported in the last symposium. In our recent study further investigation of the possible pathway from the stimulated unilateral VL nucleus to bilateral cortex and some analysis of augmenting response were carried out with 50 parkinsonism patients. The impulse which provokes the first positive wave of the contralateral side was thought to be transmitted from stimulated VL nucleus to the contralateral cortex directly via corpus callosum with a velocity of $36-43 \mathrm{~m} / \mathrm{sec}$. Repetitive stimulation of the VL nucleus showed typical augmenting or recruiting-like augmenting responses under a certain condition. 